\title{
Clinical pharmacogenomics of carvedilol: the stereo-selective metabolism angle
}

\author{
Barbara M Parker ${ }^{1}$, Sara L Rogers² \& Anastasios Lymperopoulos*,1 \\ ${ }^{1}$ Laboratory for the Study of Neurohormonal Control of the Circulation, Department of Pharmaceutical Sciences, Nova \\ Southeastern University College of Pharmacy, Ft. Lauderdale, FL 33328, USA \\ ${ }^{2}$ American Society of Pharmacovigilance, Houston, TX 77225, USA \\ *Author for correspondence: Tel.: + 1954262 1338; Fax: + 1954262 2278; al806@nova.edu
' 6 the three major enzymes/proteins carrying out carvedilol's stereo-selective metabolism are the highly polymorphic CYP2D6, UGT1 and P-glycoprotein."”

First draft submitted: 12 July 2018; Accepted for publication: 12 July 2018; Published online: 8 August 2018

\begin{abstract}
Keywords: $\beta$-blocker • carvedilol • CYP2D6 • drug interactions • enantiomer • heart failure • P-glycoprotein - pharmacokinetics • polymorphism • stereo-selective metabolism
\end{abstract}

Carvedilol is an adrenergic receptor (AR) antagonist blocking several different AR types. Specifically, it acts as an inverse agonist at the $\beta_{1}$ - and $\beta_{2}$-ARs, as well as blocks $\alpha_{1}$ ARs in the vasculature, thereby causing vasodilatation [1]. In the heart, it is relatively $\beta_{1} A R$-specific, since this AR type is by far the most predominant one in the adult human myocardium (both failing and nonfailing) [2]. Since it blocks almost all ARs in the body (with the exception of $\left.\alpha_{2} A R s\right)$, it can counter the deleterious effects of the sympathetic hyperstimulation that accompanies and aggravates chronic heart failure (CHF) [1,2]. Thus, it is used clinically with great success to treat CHF, hypertension, coronary artery disease and cardiac arrhythmias [1]. Recent studies indicate that its cardio-protective effects might be useful also in cancer chemotherapy, which oftentimes is cardiotoxic [3]. Carvedilol is very lipophilic undergoing mainly biliary elimination by the liver [1]. Importantly, the clinically used drug is a racemic mixture, with the $S(-)$ enantiomer having much higher affinity for the cardiac $\beta_{1}$ ARs and $\beta_{2} A R s$ (in addition to its affinity for the $\alpha_{1}$ ARs) than the $R(\mathcal{C})$ enantiomer, which is essentially a pure $\alpha_{1}$ AR-blocker [2]. This is crucial not only for the pharmacodynamic effects of this third generation $\beta$-blocker but also for its pharmacokinetics. Orally administered carvedilol undergoes extensive stereo-selective hepatic first-pass metabolism, resulting in almost double maximal plasma concentration (and bioavailability) of $R$-carvedilol compared with that of $S$-carvedilol [1]. Carvedilol is metabolized via aromatic ring and side-chain hydroxyl group oxidative Phase I reactions, mediated mainly by CYP2D6 (but also, to a lesser extent, CYP2C9). These are followed by Phase II conjugating reactions (glucuronidation, hydrophilic for renal; sulfation, lipophilic for hepatic/biliary tract elimination) [1]. Although carvedilol's metabolism by CYP1A2, CYP2C9, CYP2D6 and CYP3A4 is clearly documented, whether other CYP enzymes participate is currently unclear [1]. Carvedilol is a substrate for uridine diphosphate glucuronosyltransferase $1 \mathrm{~A} 1,-2 \mathrm{~B} 4$ and $-2 \mathrm{~B} 7$ and is metabolized in hepatic microsomes, as well [4]. Finally, P-glycoprotein, encoded by the MDR1 gene binds carvedilol with high affinity and affects bioavailability [1]. Given that almost all these metabolizing enzymes display significant genetic variation in humans and some CYP enzymes display carvedilol enantiomer-selectivity, for example, CYP2D6 metabolizes $S$-carvedilol much more than $R$-carvedilol [1], it becomes clear that carvedilol's pharmacokinetics is a very complex issue factoring into the drug's clinical use.

In human liver microsomes, CYP2D6 appears mainly responsible for metabolizing $R$-carvedilol, followed by CYP3A4, CYP1A2 and CYP2C9 [5]. The $S$-enantiomer is primarily metabolized by CYP1A2 followed by CYP2D6 and CYP3A4 [5]. Thus, not only CYP2D6 but also CYP1A2 and CYP3A4 carry out the stereo-selective metabolism of carvedilol in humans. Nonetheless, plasma concentrations of carvedilol upon chronic treatment can be predicted by CYP2D6 genotyping in healthy subjects, as well as by intestinal P-glycoprotein and its close relative, multidrug resistance protein MRP-2 levels [1]. The human CYP2D6 gene is highly polymorphic with over 70 alleles and 130 sequence variations [1]. This gives rise to four phenotypic groups: poor metabolizers (PMs) with no functional alleles 
at all (5-10\% frequencey in Caucasians, rare in Asians and Africans [1]); intermediate metabolizers (IMs), with one null or two deficient alleles, most commonly $C Y P 2 D 6^{*}$ ), ${ }^{*} 10,{ }^{*} 17$ and ${ }^{*} 41$ (up to $50 \%$ of Asians carrying most commonly $C Y P 2 D 6^{*} 10$ and up to $30 \%$ of Africans carrying usually $C Y P 2 D 6^{*} 17$ [1]); extensive metabolizers, the normal CYP2D6 phenotype, with two wild-type CYP2D6 alleles and normal enzymatic activity (60-85\% frequency in Caucasians [1]) and ultra-rapid metabolizers with more than two CYP2D6 copies due to gene duplication (1-10\% of Caucasians [1]). As far as carvedilol is concernced, a study in healthy Japanese subjects reported that oral clearance of both carvedilol enantiomers was significantly lower in $C Y P 2 D 6^{*} 10$ allele carriers than in $C Y P 2 D 6^{*} 1 /{ }^{*} 1,{ }^{*} 1{ }^{*} 2$ or ${ }^{*} 2{ }^{*} 2$ genotype carriers [6]. On the other hand, CYP2C9*3, CYP2C19*2, CYP2C19*3, CYP3A5*3, UGT2B $7^{*} 2$ and MDR1 C3435T did not significantly affect the pharmacokinetics of carvedilol in Japanese subjects [6]. The increased bioavailability of carvedilol observed in Japanese IM CYP $2 D 6^{*} 10$ carriers was attributed to both reduced hepatic first-pass metabolism and decreased systemic clearance [6]. Therefore, carvedilol is often undermetabolized in Japanese patients due to the high frequency of the IM phenotype-imparting CYP2D6*10 polymorphism, which overshadows the extreme rareness of the CYP2D6 PM phenotype in this population [6]. Accordingly, retrospective analysis of carvedilol's side effects in clinical trials showed that CYP2D6 PMs had a higher rate of dizziness during dose titration, presumably resulting from enhanced vasodilating effects of carvedilol [7], whose enantiomers are both $\alpha_{1}$ AR-blockers [1,2].

$S$-carvedilol also undergoes higher, compared with $R$-carvedilol, glucuronidation in human liver and intestinal microsomes, similar to the levels of CYP2D6-dependent oxidation of the two enantiomers [8]. As already mentioned, this stereo-selective metabolism of carvedilol is carried out mainly by CYP2D6 but also by CYP1A2 in the liver and by CYP3A4 in the gut [8]. Thus, not only the oxidation but also the subsequent UGT1A-dependent glucuronidation of carvedilol in intestinal epithelial cells is stereo-selective in humans [8], with both metabolic processes (oxidation and glucuronidation) proceeding at a higher rate for the $S$ - than for the $R$-enantiomer, resulting in more $S$ carvedilol's presystemic clearance. This may translate into more pronounced carvedilol-induced vasodilatation (manifested clinically as orthostatic hypotension) than cardio-suppression. Intriguingly, the two enantiomers have been shown to even inhibit each other's glucuronidation through UGT1A1, UGT2B4 and UGT2B7 in human liver microsomes [9]. UGT2B7 shows a preference for $S$-carvedilol's metabolism, whereas $R$-carvedilol is metabolized equally by these three UGT isoforms [9]. Additionally, PM-associated polymorphisms in human UGT genes $\left(U G T 1 A 1^{*} 6\right.$ and $\left.U G T 2 B 7^{*} 3\right)$ are linked with poor glucuronidation of both enantiomers in Japanese subjects [1]. The mutual metabolic inhibition of the two enantiomers at the level of glucuronidation presents another obstacle in the effort to predict carvedilol's clinical bioavailability.

Finally, carvedilol is a known P-glycoprotein ligand [1]. P-glycoprotein negatively affects the drug's intestinal absorption. More importantly however, carvedilol, like two other clinically useful $\beta$-blockers, propranolol and bisoprolol, is a potent inhibitor of this plasma membrane transporter [10]. This leads to several, potentially significant drug-drug interactions of carvedilol, such as with cyclosporine and digoxin, two major P-glycoprotein substrates $[10,11]$. The interaction with digoxin might be more pronounced in men than in women, given the higher expression/activity of P-glycoprotein in males [11]. P-glycoprotein inhibition by carvedilol may also increase doxorubicin's cytotoxicity, although this is probably insignificant clinically, since P-glycoprotein inhibition can boost chemotherapeutic drug levels offsetting this and carvedilol exerts significant protective effects against chemotherapy-induced cardiotoxicity in its own right $[3,12]$. Indeed, carvedilol-induced P-glycoprotein blockade does not affect the in vivo intestinal absorption of clopidogrel to a significant extent and carvedilol does not seem to have a clinically relevant interaction with this common antiplatelet drug, which is also a P-glycoprotein substrate [13]. Of note, metoprolol, one of the three $\beta$-blockers currently US FDA-approved for human CHF treatment (the other two being carvedilol and bisoprolol), lacks inhibitory effects on P-glycoprotein [10]. This distinguishes metoprolol from both carvedilol and bisoprolol and suggests that metoprolol may be the $\beta$-blocker of choice in CHF when P-glycoprotein-dependent drug interactions are a clinical concern, for example, upon concomitant digoxin or cyclosporine treatment.

Unfortunately, there is a paucity of studies assessing the utility of carvedilol's stereo-selective pharmacokinetics for integration into clinical practice, whether pertaining to clinical efficacy or to need for dose adjustments due to drugdrug interactions. The very limited data available to date suggest that no dose adjustment is necessary in diabetic patients, despite the potential inhibition of carvedilol's CYP2C9-dependent metabolism by oral hypoglycemic agents like glibenclamide [14]. This is probably due to the higher CYP2D6-mediated metabolism of carvedilol compensating for the blocked CYP2C9-dependent elimination [14]. Additionally, in portal hypertension, one of the major therapeutic indications of carvedilol, the drug's efficacy against liver fibrosis is unaltered, despite delayed 
clearance due to reduced hepatic blood flow and CYP2D6 expression levels [15]. This was attributed to the overall improvement of hepatic function and blood flow by the hepatic pharmacodynamic effects of carvedilol, which ultimately restored/enhanced the drug's own pharmacokinetics, as well [15]. A study in Japanese CHF patients found that the commonly used antiarrhythmic drug amiodarone can selectively inhibit $S$-carvedilol's metabolism, resulting both in a higher concentration-to-dose ratio for $S$-carvedilol and in a higher $S$-to- $R$ enantiomer ratio in patients co-administered with the two drugs [16]. Given that the $R$ enantiomer, whose metabolism is unaffected by amiodarone, essentially bocks $\alpha_{1}$ ARs only while the $S$ enantiomer is the cardiac $\beta$ AR blocker (see above), the interaction of carvedilol with amiodarone may predispose to severe bradycardia and cardiac depression, especially in CHF patients, whose cardiac $\beta_{1}$ AR levels are significantly downregulated [2]. Studies in Japanese CHF populations have clearly indicated that both enantiomers undergo lower metabolism in CHF compared with healthy subjects, with $R$-carvedilol being metabolized significantly less than $S$-carvedilol [17]. In addition, their metabolism in CHF is dependent on CYP2D6 genotype, $\alpha(1)$-acid glycoprotein (an $\alpha$ globulin of plasma with a very similar to Pglycoprotein drug-binding profile) and bodyweight [17]. It is also dependent on the type of formulation of oral carvedilol administered, in other words, immediate-(IR) versus controlled-release (CR) [18]. The enantiomers of CR-carvedilol have a longer half-life and slower overall metabolism than those of IR-carvedilol. On the other hand, a study in Israeli CHF patients found no significant differences in carvedilol dosages or rates of adverse drug reactions (ADRs) among CYP2D6 phenotypic groups, despite a similar CYP2D6 phenotypic distribution to the one in the European general population [19]. This study concluded that CYP2D6 genotype and number of ADRs were not predictive of carvedilol dose changes in CHF patients [19]. Similarly, a study by Sehrt et al. in 2011 in European (German) subjects found no effect of the CYP2D6 genotype on heart rate, blood pressure or ADRs upon carvedilol administration (reviewed in [1]). Instead, it uncovered associations of $\beta$ AR gene polymorphisms ( $\beta_{1}$ AR Gly 49 and $\beta_{2} \mathrm{AR}$ Gln27) with greater reductions in exercise heart rates and resting blood pressure by carvedilol, in other words, it found genetic associations with the pharmacodynamics, rather than with the pharmacokinetics of the drug. In addition to the different ethnicities involved, another reason for the discrepancy between this and the Japanese studies discussed above may very well be the fact that the German study was done in healthy volunteers and not in CHF patients, in whom carvedilol's pharmacokinetics are significantly altered $[1,17]$. Nevertheless, this European study showcases the significant effect AR pharmacogenetics (for example, $\beta_{1}$ AR Gly49Ser and Arg389Gly; $\beta_{2}$ AR Arg16Gly and Gln27Glu) can have on carvedilol's pharmacodynamic responses in humans [20]. However, this is beyond the scope of the present article and will not be discussed any further here.

In summary, multiple variables complicate interpretation of the available studies regarding effects of the stereoselective metabolism of carvedilol on the drug's patient response. Apart from being very few and commonly ridden with limited statistical power, the available studies differ in ethnicity, age, sex, bodyweight and co-morbidities of patient populations, in the formulation and/or delivery form of carvedilol used (immediate vs controlled release; oral vs parenteral) and some are conducted in healthy volunteers as opposed with other studies done in CHF patients. Another layer of complexity is added by the complicated pharmacodynamics of the drug, in other words, the multitude of its target receptors, which are also enantiomer-selective and can offset some of the effects of carvedilol's stereo-selective metabolism. Nevertheless, some conclusions regarding the contribution of carvedilol's stereo-selective metabolism to the drug's overall clinical pharmacogenomics can still be drawn. Thus, it appears that the three major enzymes/proteins carrying out carvedilol's stereo-selective metabolism are the highly polymorphic CYP2D6, UGT1 and P-glycoprotein. However, P-glycoprotein genotype seems to affect interactions of carvedilol with co-administered drugs only (for example, digoxin and cyclosprorine). Therefore, when another strong P-glycoprotein substrate is administered concomitantly with carvedilol, care must be taken to properly adjust the second drug's dose or to substitute carvedilol with the equally effective metoprolol that lacks Pglycoprotein-dependent drug-drug interactions. Additionally, carvedilol's inhibitory effects on P-glycoprotein may be of value in enhancing cancer chemotherapy's efficacy in clinical oncology, especially since carvedilol per se is also cardio-protective [3]. Unlike P-glycoprotein, CYP2D6 and UGT1 genotypes do affect carvedilol's stereo-selective metabolism. Both of these enzymes metabolize carvedilol with a stereo-selective preference for the $S$-enantiomer. Given that this is essentially the $\beta$-blocker component of carvedilol, CYP2D6 and UGT1 pharmacogenetics may have significant impact on patient response. Indeed, at least in Japanese CHF patients, an ethnic group with high prevalence of the CYP2D6 IM phenotype, levels of the vasodilatory $R$-carvedilol may prevail over those of the $\beta$ AR-blocking $S$-enantiomer, which can predispose to arrhythmias and to carvedilol's therapeutic failure, as cardiac sympathetic hyperstimulation may get incompletely buffered by the orally administered carvedilol. Even in that case however, prediction of the ultimate effect on patient's response is very risky, since the complex pharmacodynamics of 
carvedilol may offset this effect of its stereo-selective metabolism. More specifically, whatever vasodilation-induced, baroreflex-mediated cardiac stimulation the higher $R$-carvedilol (than $S$-carvedilol) levels may impart could get counterbalanced by the low cardiac $\beta_{1}$ AR density of the CHF patient; this receptor is selectively downregulated in human failing hearts and carvedilol, paradoxically, does not upregulate it like the other $\beta$-blockers do [2]. On the other hand, the low prevalence of CYP2D6 PM and IM phenotypes in non-Asian populations probably accounts, in large part, for the discrepancies between European/Israeli and Japanese studies on the contributions of CYP2D6 phenotypes to carvedilol's clinical pharmacogenomics.

In conclusion, carvedilol's large therapeutic index preempts any significant impact of its stereo-selective metabolism by the polymorphic CYP2D6 and UGT enzymes on its safety or ADR propensity. In all likelihood, the only parameter that carvedilol's stereo-selective metabolism can possibly affect is the risk for therapeutic failure in CHF or hypertensive patients. However, it is virtually impossible to gauge this therapeutic failure risk due to the plethora of carvedilol's formulations/delivery forms and its complex pharmacodynamics. This notwithstanding, it can be concluded from the above reviewed studies that whenever a dose adjustment decision for carvedilol is needed, the drug's enantiomer-selective metabolism has to be taken into account, especially if the patient is of Asian ethnicity and has CHF. More studies with sufficient statistical power in Caucasian, Hispanic and black CHF patient populations are definitely warranted to assign a predictive value of pharmacodynamic response (or lack thereof) to carvedilol's stereo-selective and genetically polymorphic metabolism.

\section{Financial \& competing interests disclosure}

The authors have no relevant affiliations or financial involvement with any organization or entity with a financial interest in or financial conflict with the subject matter or materials discussed in the manuscript. This includes employment, consultancies, honoraria, stock ownership or options, expert testimony, grants or patents received or pending, or royalties.

No writing assistance was utilized in the production of this manuscript.

\section{References}

1. Lymperopoulos A, McCrink KA, Brill A. Impact of CYP2D6 genetic variation on the response of the cardiovascular patient to carvedilol and metoprolol. Curr. Drug Metab. 17(1), 30-36 (2015).

2. Yoshikawa T, Port JD, Asano K et al. Cardiac adrenergic receptor effects of carvedilol. Eur. Heart J. 17(Suppl. B), 8-16 (1996).

3. Avila MS, Ayub-Ferreira SM, de Barros Wanderley MR Jr et al.Carvedilol for prevention of chemotherapy: related cardiotoxicity: the CECCY trial. J. Am. Coll. Cardiol. 71(20), 2281-2290 (2018).

4. Ohno A, Saito Y, Hanioka N et al. Involvement of human hepatic UGT1A1, UGT2B4 and UGT2B7 in the glucuronidation of carvedilol. Drug Metab. Dispos. 32(2), 235-239 (2004).

5. Iwaki M, Niwa T, Bandoh S et al. Application of substrate depletion assay to evaluation of CYP isoforms responsible for stereoselective metabolism of carvedilol. Drug Metab. Pharmacokinet. 31(6), 425-432 (2016).

6. Honda M, Ogura Y, Toyoda W et al. Multiple regression analysis of pharmacogenetic variability of carvedilol disposition in 54 healthy Japanese volunteers. Biol. Pharm. Bull. 29(4), 772-778 (2006).

7. Coreg, package insert. GlaxoSmith Kline, NC, USA.

8. Hanioka N, Tanaka S, Moriguchi Y, Narimatsu S. Stereoselective glucuronidation of carvedilol in human liver and intestinal microsomes. Pharmacology 90(3-4), 117-124 (2012).

9. Takekuma Y, Yagisawa K, Sugawara M. Mutual inhibition between carvedilol enantiomers during racemate glucuronidation mediated by human liver and intestinal microsomes. Biol. Pharm. Bull. 35(2), 151-163 (2012).

10. Bachmakov I, Werner U, Endress B, Auge D, Fromm MF. Characterization of $\beta$-adrenoceptor antagonists as substrates and inhibitors of the drug transporter P-glycoprotein. Fundam. Clin. Pharmacol. 20(3), 273-282 (2006).

11. Baris N, Kalkan S, Güneri S, Bozdemir V, Guven H. Influence of carvedilol on serum digoxin levels in heart failure: is there any gender difference? Eur. J. Clin. Pharmacol. 62(7), 535-538 (2006).

12. Takara K, Sakaeda T, Okumura K. Carvedilol: a new candidate for reversal of MDR1/P-glycoprotein-mediated multidrug resistance. Anticancer Drugs 15(4), 303-309 (2004).

13. Incecayir T, Ilbasmis-Tamer S, Tirnaksiz F, Degim T. Assessment of the potential drug-drug interaction between carvedilol and clopidogrel-mediated through intestinal P-glycoprotein. Pharmazie 71(8), 472-477 (2016).

14. Nardotto GHB, Lanchote VL, Coelho EB, Della Pasqua O. Population pharmacokinetics of carvedilol enantiomers and their metabolites in healthy subjects and Type-2 diabetes patients. Eur. J. Pharm. Sci. 109S, S108-S115 (2017).

15. El-Demerdash E, Abdel-Sattar SA, El-Bakly WM, Mohamed EA. Antifibrotic effects of carvedilol and impact of liver fibrosis on carvedilol pharmacokinetics in a rat model. Eur. J. Drug Metab. Pharmacokinet. 42(5), 767-779 (2017). 
16. Fukumoto K, Kobayashi T, Komamura K, Kamakura S, Kitakaze M, Ueno K. Stereoselective effect of amiodarone on the pharmacokinetics of racemic carvedilol. Drug Metab. Pharmacokinet. 20(6), 423-427 (2005).

17. Saito M, Kawana J, Ohno T et al. Population pharmacokinetics of R-and S-carvedilol in Japanese patients with chronic heart failure. Biol. Pharm. Bull. 33(8), 1378-1384 (2010).

18. Packer M, Lukas MA, Tenero DM, Baidoo CA, Greenberg BH, 369 Study Group. Pharmacokinetic profile of controlled-release carvedilol in patients with left ventricular dysfunction associated with chronic heart failure or after myocardial infarction. Am. J. Cardiol. 98(7A), 39L-45L (2006).

19. Shihmanter R, Nulman I, Goland $S$ et al.Variation in the CYP2D6 genotype is not associated with carvedilol dose changes in patients with heart failure. J. Clin. Pharm. Ther. 39(4), 432-438 (2014).

20. Ahles A, Engelhardt S. Polymorphic variants of adrenoceptors: pharmacology, physiology and role in disease. Pharmacol. Rev. 66(3), 598-637 (2014). 
